J. Clin. Chem. Clin. Biochem.

Vol. 16, 1978, pp. 335-342

\title{
Bestimmung von Digoxin im Serum \\ Vergleich von Radioimmunassay und heterogenem Enzymimmunassay
}

\author{
Von K. Borner \\ Institut für Klinische Chemie und Klinische Biochemie der Freien Universität Berlin, Klinikum Steglitz und \\ N. Rietbrock \\ Institut für Klinische Pharmakologie der Freien Universität Berlin, Klinikum Steglitz
}

(Eingegangen am 7. November 1977/16. Februar 1978)

Herrn Professor Dr. Dr. Ernst Schütte zum 70. Geburtstag gewidmet

Zusammenfassung: Es werden erste praktische Erfahrungen mit einem heterogenen Enzymimmunassay (EIA, Typ ELIS 4) ${ }^{1}$ ) zur Bestimmung von Digoxin im Serum berichtet und mit den Ergebnissen eines Radioimmunassays (New England Nuclear, ${ }^{125} \mathrm{~J}$-Digoxin) verglichen. Die Nachweisgrenze beträgt etwa 0,3 $\mu \mathrm{g} / 1(0,4 \mathrm{nmol} / \mathrm{l})$. Der Meßbereich erstreckt sich von 0,3 bis $5,0 \mu \mathrm{g} / 1(0,4$ bis 6,4 nmol/l). Die Präzision in Serie liegt bei 7-11\% und die Präzision von Tag zu Tag bei 8-14\%. Zu Albumin-Lösungen und Pool-Serum zugesetztes Digoxin wurde zu 87 bis $106 \%$ wiedergefunden. 116 Serumproben von Patienten, die ausschließlich Digoxin einnahmen, ergeben im Vergleich zum Radioimmunassay (RLA) keinen systematischen Unterschied. Gleichung der mittleren Regressionsgeraden:

$\mathrm{C}_{\mathrm{ELISA}}=0,954 \cdot \mathrm{C}_{\mathrm{RIA}}+0,14(\mu \mathrm{g} / 1 \mathrm{bzw} \cdot 0,18 \mathrm{nmol} / \mathrm{l})$. Jedoch bestehen im Einzelfall merkliche Differenzen. Digitoxin ergibt in vivo und in vitro je nach Konzentration eine Kreuzreaktion von 7 bis $14 \%$. Bei 68 Patienten, die zusätzlich Spironolacton einnahmen, ergab der Enżymimmunassay durchschnittlich 0,48 $\mu \mathrm{g} / 1(0,64 \mathrm{nmol} / \mathrm{l})$ höhere Ergebnisse als der Radioimmunassay (Bereich 0,14 bis $1,14 \mu \mathrm{g} / \mathrm{l}$ bzw. 0,18 bis $1,46 \mathrm{nmol} / \mathrm{l} ; \mathrm{p}<0,01 \%$ ). In Bezug auf Praktikabilität entspricht der ELISA weitgehend dem analogen Radioimmunassay mit solid phase technique.

\section{Determination of digoxin in serum. Comparison of radioimmunoassay and a heterogeneous enzyme immunoassay}

Summary: This paper describes the evaluation of a heterogeneous enzymeimmunoassay (EIA, subtype ELISA) for the determination of digoxin in serum. Results are compared with those obtained from a radioimmunoassay (New England Nuclear, ${ }^{125} \mathrm{I}$-Digoxin). The limit of detection is $0.3 \mu \mathrm{g} / 1(0.4 \mathrm{nmol} / \mathrm{l})$. The range of the test is from 0.3 unto $5.0 \mu \mathrm{g} / \mathrm{l}$ (0.4-6.4 nmol/1). Within-batch precision ranged from 7 to $11 \%$, between-batch precision from $8-14 \%$. Pure digoxin, added to solutions of albumin and pooled sera, gave recoveries between 87 and $106 \% .116$ sera from patients taking digoxin exclusively yielded no systematic difference compared to the results of a radioimmunoassay (RIA) (equation of the bivariate regression: $C_{\text {ELISA }}=0.954 \cdot \mathrm{C}_{\mathrm{RIA}}+0.14(\mu \mathrm{g} / \mathrm{l})(0.18 \mathrm{nmol} / \mathrm{l})$. Nevertheless marked differences between both tests were observed in individual cases. Digitoxin yielded in vitro and in vivo a cross-reactivity of 7 to $14 \%$ depending upon the concentration. In 68 sera from patients, taking digoxin plus spironolactone, we found results that were on average $0.48 \mu \mathrm{g} / 1(0.64 \mathrm{nmol} / \mathrm{l})$ higher with the ELISA than with the RIA $(\mathrm{p}<0.01 \%)$. With respect to practicability the ELISA test for digoxin is very similar to the analogous RIA both using the solid phase technique.

\section{Einfuihrung}

Die regelmäßige Kontrolle der Șerum-Konzentration von Digoxin hat die Therapie mit Digoxin sicherer und zuverlïssiger gemacht $(1,2)$. An eine dafür geeignete Analysenmethode werden hohe Anforderungen in Bez̈ug auf Nachweis-Empfindlichkeit und Spezifität gestellt. Bis
Ende 1975 war der Radioimmunassay (RIA) die einzige Methode, die praktikabel genug war, für klinische RoutineŻwecke eingesetzt werden zu können (3). Der den Einsatz des Radioimmunassay stark limitierende Faktor war die Notwendigkeit des Umgangs mit radioaktivem Material. Mit dem Aufkommen von enzym-markierten Liganden wurden Immunassays vorgeschlagen (Übersicht 
bei 1.c. (4)), die diesen Nachteil nicht aufweisen. Beim Enzymimmunassay ersetzt ein Enzym den radioaktiven Indikator (engl. marker). So entwickelten Chang et al. (5) einen homogenen Enzymimmunassay zur Bestimmung von Digoxin, über dessen Anwendung inzwischen mehrfach berichtet wurde (6-10). Alternativ dazu wird seit kurzem ein heterogener Enzymimmunassay zur Bestimmung von Digoxin angeboten (11), der die sog. solid phase technique des Radioimmunassay verwendet, die von ihren Beschreibern das Akronym ELISA (enzyme linked immunosorbent assay) ${ }^{1}$ ) erhielt (12).

Der Test besteht aus 3 Schritten:

1. Konkurrierende Bindung von freiem Digoxin und enzymmarkiertem Digoxin an den wandständigen Antikörper.

2. Trennen von gebundenem und freiem Hapten-Enzym durch Dekantierten und

3. Bestimmen der Antikörper-gebundenen, wandständigen Enzymaktivität.

Der hier geprüfte Test verwendet Peroxidase (EC 1.11.1.7) als Indikator. Die vorliegende Arbeit berichtet über praktische Erfahrungen mit der Bestimmung von Digoxin im Serum durch einen heterogenen Enzymimmunassay im Vergleich zu einem in der Praxis bewährten Radioimmunassay. Dabei sollte geklärt werden, ob der heterogene Enzymimmunassay den Radioimmunassay in Bezug auf Zuverlässigkeit und Praktikabilität erreicht.

\section{Material und Methoden}

Klinische Proben

Verwendet wurden Seren von Patienten des Klinikums Steglitz und von externen Einsendern. Die Blutabnahme erfolgte frühestens 12 Stunden nach der letzten Medikamenteneinnahme. Die Proben wurden in der Regel am Tage der Entnahme verarbeitet.

Reagenzien und Bestimmungsmethoden Alle RIA-Bestimmungen wurden doppelt, die ELISA-Bestimmungen dreifach durchgeführt.

\section{Radioimmunassay von Digoxin}

Test RIA-NEN Digoxin der Fa. New England Nuclear (North Billerica, Mass.) Prinzip: ${ }^{125} \mathrm{~J}$-Markierung von Digoxin, Solid phase assay system.

\section{Radioimmunassay von Digitoxin}

$125 \mathrm{~J}$ Gamma coat digitoxin der Fa. Clinical Assays (Cambridge, Mass.). Prinzip: ${ }^{125} \mathrm{~J}$-Markierung von Digitoxin, Solid phase assay system.

1) Im Text verwendete Abkürzungen:

ELISA enzyme linked immuno sorbent assay

RIA radioimmunoassay
Heterogener Enzymimmunassay zur Digoxin-Bestimmung. Enzymun-Test Digoxin, Best. Nr. 199656, Fa. Boehringer (Mannheim). Chargen Nummern 1046504, 1186307, 1256108.

Analytisches Verfahren des heterogenen Enzymimmunassays

1. Kompetitive Bindung

$0,1 \mathrm{ml}$ Standard/Serum/Kontrollserum

$1,0 \mathrm{ml}$ Reaktionsgemisch (Phosphatpuffer Peroxidase-markiertes Digoxin)

Inkubation in Antikörper-beschichteten Röhrchen über 60 Minuten bei Raumtemperatur.

2. Trennung von gebundenem und freiem Indikator: Absaugen des Inkubationsgemisches und Waschen der Röhrchen.

3. Indikatorreaktion der Antikörper-gebundenen Peroxidase: Füllen der Röhrchen mit 1 ml Puffer-Substratgemisch (Perborat und 2,2'-Azino-dil 3-e thyl-benzthiazolinsulfonsäure (6) ], ABTS)

Inkubation 60 min bei $25^{\circ} \mathrm{C}$.

4. Messen der Extinktion des Inkubationșgemisches bei $405 \mathrm{~nm}$.

5. Die Auswertung der Ērgebnisse erfolgte mittels gezeichneter Bezugskurven.

\section{Glykoside}

Digoxin, Fa. Merck (Darmstadt), Nr. 3047, Digitoxin, Fa. Merck (Darmstadt), Nr. 3044.

\section{Kontrollseren}

Firmen Boehringer, Dade und New England Nuclear.

\section{Meßgeräte}

Elektronischer Dilutor (Firma Brand, Wertheim).

Digitalphotometer Eppendorf 6115S (Eppendorf Gerätebau, Hamburg) mit Absaugküvette und Drucker 6522.

Szintillationsspektrometer Autogamma 5130 (Packard Instruments, Darmstadt).

\section{Statistik}

Standard-Methoden (13).

Bivariate Regressionsanalyse (14).

\section{Ergebnisse}

Bezugskurven, Meßbereich und Nachweisgrenze Abbildung 1 zeigt eine typische Bezugskurve ohne Skalentransformation. In der Anfangszeit der Erprobung wurden gelegentlich auch angedeutet sigmoide Bezugskurven ermittelt. Bei İnhịbierung des Enżyms mit Natriumazid erreicht die Kurve fast die Absorption 0, gemessen gegen einen stabilen Reagenzienleerwert von $A=0,600$ bis 0,800 . Die Nachweisgrenże beträgt nähèrungsweise $0,3 \mu \mathrm{g} / \mathrm{l}(0,4 \mathrm{nmol} / 1)$. Der von Meßpunkten eingeschlossene Bereich reicht von 0,75 bis $5 ; 0 \mu \mathrm{g} / \mathrm{l}$, in neueren Packungen von 0,3 bis $5,0 \mu \mathrm{g} / 1(0,38$ bis $6,40 \mathrm{nmol} / \mathrm{l})$. Eine reziproke Darstellung $(1 / \mathrm{A}=\mathrm{f}(\mathrm{c}))$ der Abbildung 1 ergibt eine aufwặrts gekrümmte Kurve. 


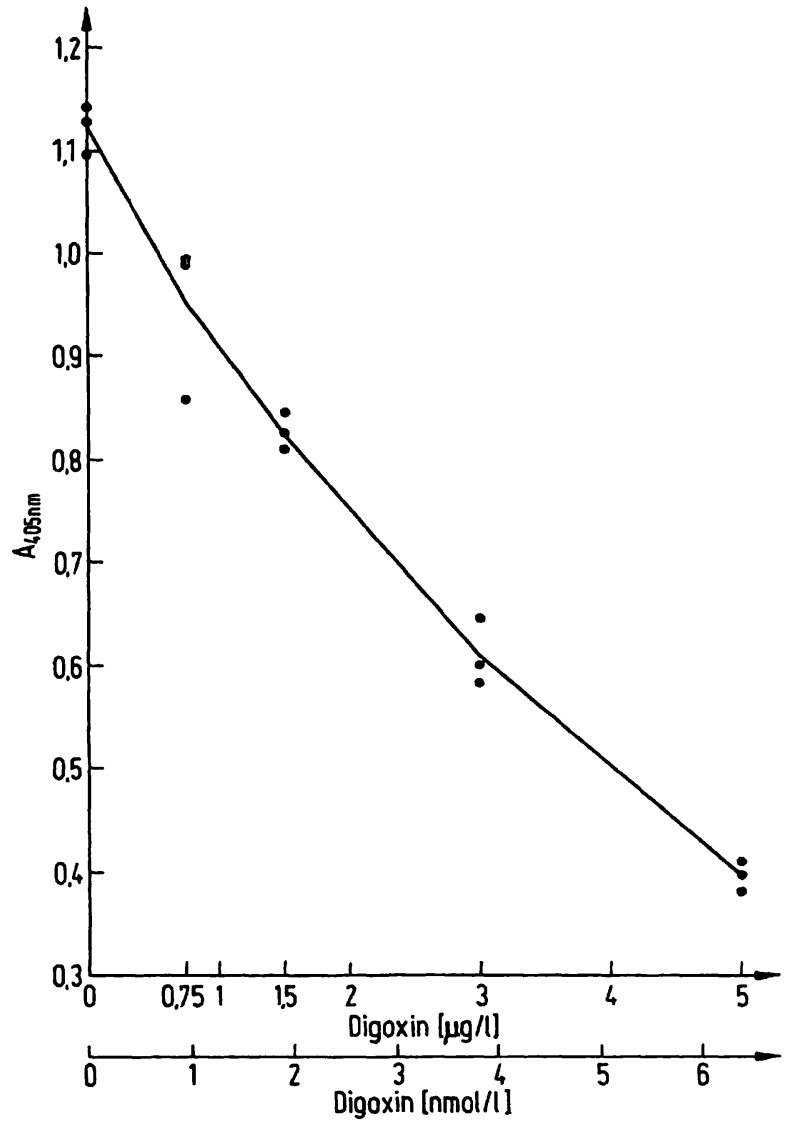

Abb. 1. Bezugskurve für Digoxin-ELISA.

\section{Präzision}

Tabelle 1 gibt einen Uberblick über die Präzision in Serie des ELISA. Gleichzeitig wurde die Präzision in Serie mit dem RIA zu Vergleichszwecken bestimmt. Die Variationskoeffizienten liegen zwischen 3 und $11 \%$.

Die Präzision von Tag zu Tag lag für den ELISA zwischen 8 und $14 \%$ und mit den gleichen Kontrollproben für den RIA bei 9 bis $15 \%$ (Tab. 2).

Dabei ist jedoch zu beachten, daß beim ELISA jeweils Dreifachbestimmungen, beim RIA jedoch nur DoppelBestimmungen ausgeführt wurden.

\section{Richtigkeit}

Die Uberprüfung der Methode mit Kontrollseren ergab die in Tabelle 2 angegebenen Werte. Zum Vergleich enthält Tabelle 2 ebenfalls die mit dem RIA gefundenen Ergebnisse.

Die für den ELISA vom Hersteller gelieferten Kalibrierlösungen ergaben mit dem RIA innerhalb der Fehlerbreite übereinstimmende Ergebnisse (Tab. 3).

Ein Zusatz von Digoxin zu einem Serumgemisch mit niedriger Digoxin-Konzentration ergab bei Zusatz von 1 bis $3 \mu \mathrm{g} / 1$ (1,28 bis $3,84 \mathrm{nmol} / \mathrm{l})$ Wiederfindungsanteíle von 99 bis $106 \%$ (Tab. 4).

Tab. 1. Präzision in Serie.

\begin{tabular}{llllllrr}
\hline Material & Methode & $\begin{array}{l}\text { Sollwert } \\
(\mu \mathrm{g} / \mathrm{l})\end{array}$ & $\begin{array}{l}\text { Istwert, } \overline{\mathbf{x}} \\
(\mathrm{nmol} / \mathrm{l})\end{array}$ & $\begin{array}{l}\mathrm{ng} / \mathrm{l}) \\
(\mathrm{nmol} / \mathrm{l})\end{array}$ & $\mathrm{n}$ & $\mathrm{VK}$ Serie $(\%)$ \\
\hline $\begin{array}{l}\text { Lederle } \\
\text { (Charge 2945-406) }\end{array}$ & RIA & 3,25 & $(4,16)$ & 3,61 & $(4,63)$ & 12 & 3,2 \\
$\begin{array}{l}\text { Kontrollserum } \\
\text { (RIA-NEN) }\end{array}$ & ELISA & 3,25 & $(4,16)$ & 3,01 & $(3,85)$ & 9 & 7,5 \\
Boehringer 3 & RIA & 3,00 & $(3,84)$ & 3,19 & $(4,08)$ & 20 & 2,9 \\
\hline
\end{tabular}

Tab. 2. Richtigkeit und Präzision von Tag zu Tạg.

\begin{tabular}{|c|c|c|c|c|c|c|c|}
\hline Material & Methode & $\begin{array}{l}\text { Sollwërt } \\
(\mu g / 1)\end{array}$ & (nmol/1) & $\begin{array}{l}\text { Istwert, } \bar{x} \\
(\mu \mathrm{g} / \mathrm{l})\end{array}$ & (nmol/l) & $\mathbf{n}$ & VK (\%) \\
\hline $\begin{array}{l}\text { Lederle I } \\
\text { (Charge 2945-405) }\end{array}$ & ELISA & $1,09^{+}$ & $(1,40)$ & 0,85 & $(1,09)$ & 7 & 13 \\
\hline $\begin{array}{l}\text { Lederle I } \\
\text { (Charge 2945:405) }\end{array}$ & RIA & 1,09 & $(1,40)$ & 1,04 & $(1,33)$ & 4 & 15 \\
\hline $\begin{array}{l}\text { Lederle II } \\
\text { (Charge 2945-406) }\end{array}$ & ELISA & $3,25^{+}$ & $(4,16)$ & 3,40 & $(4,36)$ & 13 & 13,7 \\
\hline $\begin{array}{l}\text { Lederle II } \\
\text { (Charge 2945-406) }\end{array}$ & $\tilde{\mathbf{R}} \mathbf{I A}$ & 3,25 & $(4,16)$ & 3,39 & $(4,34)$ & 8 & 9,2 \\
\hline Boehringer 3 & ELISA & 2,50 & $(3,20)$ & 2,53 & $(3,24)$ & 5 & 8,1 \\
\hline Boehringer 4 & ELIISA & 4,00 & $(5,12)$ & 3,90 & $(4,99)$ & 4 & 11,8 \\
\hline Boehringer 6 & ELISA & 2,50 & $(3,20)$ & 2,54 & $(3,25)$ & 14 & 11,4 \\
\hline
\end{tabular}

+ Der angegebene Sollwert wưurdẹ mit einem RIA exmittelt. 
Tab. 3. Vergleich mit Kalibrier-Lösungen.

\begin{tabular}{|c|c|c|c|}
\hline $\begin{array}{l}\text { Kalibrierlösung } \\
\text { angegebener Wert } \\
\text { Boehringer }(\mu \mathrm{g} / \mathrm{l})\end{array}$ & (nmol/l) & $\begin{array}{l}\text { mit RIA } \\
\text { gefunde } \\
(\mu g / 1)\end{array}$ & $\begin{array}{l}\text { V } \\
\text { Vert } \\
(\mathrm{nmol} / \mathrm{l})\end{array}$ \\
\hline $\begin{array}{l}0 \\
0,75 \\
1,50 \\
3,00 \\
5,00\end{array}$ & $\begin{array}{l}(0) \\
(0,96) \\
(1,92) \\
(3,84) \\
(6,40)\end{array}$ & $\begin{array}{l}0,18 \\
0,60 \\
1,38 \\
3,08 \\
5,01\end{array}$ & $\begin{array}{l}(0,23) \\
(0,77) \\
(1,77) \\
(3,94) \\
(6,41)\end{array}$ \\
\hline
\end{tabular}

Bei Zusatz von $3 \mu \mathrm{g} / \mathrm{l}(3,84 \mathrm{nmol} / \mathrm{l})$ Digoxin zu wäßrigen Albumin-Lösungen wurden 87 bis $97 \%$ der Einwaage wiedergefunden. Eine Abhängigkeit von der Albuminkonzentration $(10,20,30,40,50 \mathrm{~g} / \mathrm{l})$ besteht nicht.

\section{Methodenvergleich}

In 116 Serumproben von Patienten, die ausschließlich Digoxin einnahmen, wurde Digoxin mit dem ELISA und dem RIA bestimmt. Die Ergebnisse sind in Abbildung 2 dargestellt. Ein statistischer Vergleich beider Kollektive ergibt keinen signifikanten Unterschied (Tab. 5). Auch die mittlere Regressionsgerade weicht nur geringfügig von der Winkelhalbierenden $a b$.

\section{Spezifität}

\section{Digitoxin}

Wäßrige Lösungen von Digitoxin und in vitro mit Digitoxin versetztes Pool-Serum von 5 gesunden Personen unter 30 Jahren ergibt eine Kreuzreaktion in DigoxinELISA von 7 bis $14 \%$ (Tab. 6). Die Kreuzreaktion ist konzentrationsabhängig.

Bei Seren von Patienten, die Digitoxin einnahmen, ist die Kreuzreaktion merklich höher. Sie beträgt für den Digoxin-ELISA zwischen 12 und $40 \%$ bei DigitoxinKonzentrationen zwischen 10 und $38 \mu \mathrm{g} / 1$ (13,1 und $49,7 \mathrm{nmol} / \mathrm{l}$ ) (Tab. 7). Die zum Vergleich gleichzeitig bestimmte Kreuzreaktion des Digoxin-RIA liegt zwischen 3 und $15 \%$.

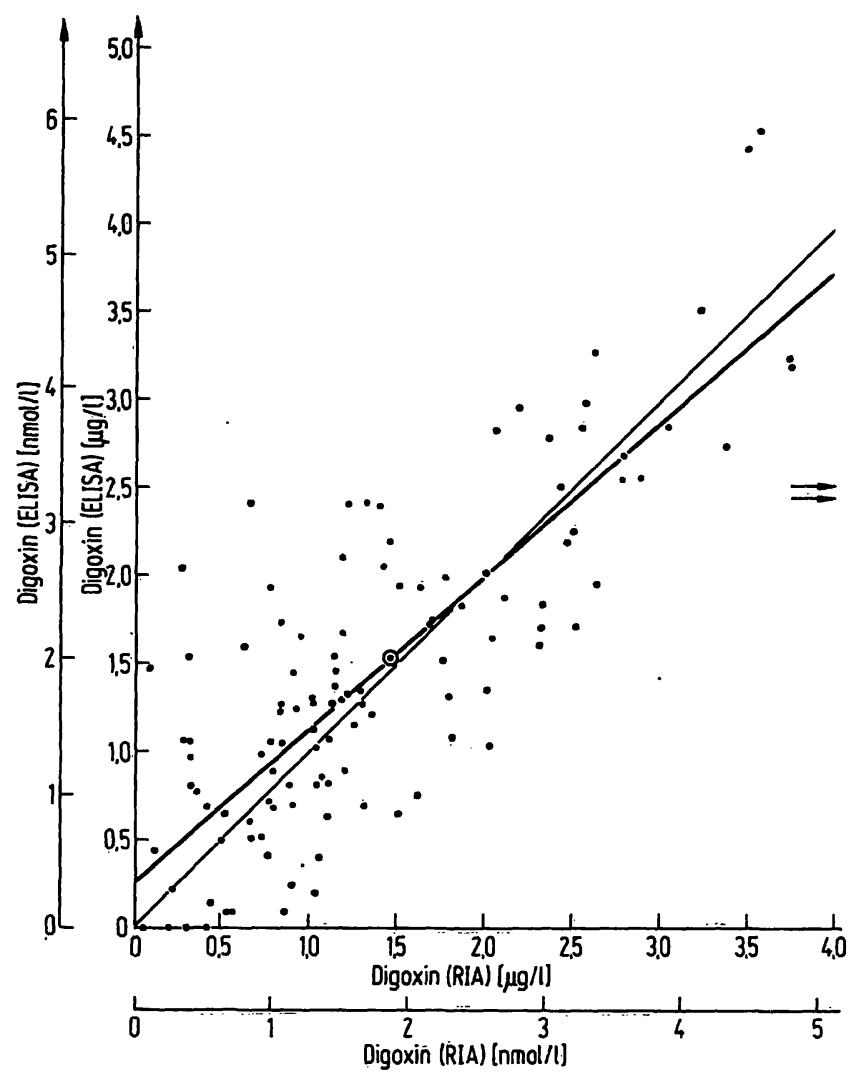

Abb. 2. Vergleich der Ergebnisse von Digoxin-Bestimmungen bei Patienten, die keine zusätzlichen Medikamente erhielten.

Dünne Linie: Winkelhalbierende.

Starke Linie: mittlere Regressionsgerade $\mathrm{N}=116$ Steigung der mittleren Regressionsgeraden $=0,954$ Achsenabschnitt $=0,139 \mu \mathrm{g} / \mathrm{l}$ $[=0,178$ $\mathrm{nmol} / 1]$

Korrelationskoeffizient $=0,810$

\section{Spironolacton}

Die Bestimmung von Digoxin in 68 Seren von Patienten, die zusätzlich Spironolacton einnahmen, ergab mit dem ELISA um durchschnittlich $0,48 \mu \mathrm{g} / 1(0,62 \mathrm{nmol} / \mathrm{l})$ höhere Ergebnisse als mit dem RIA (vgl. Abb. 3). Statistische Vergleiche żeigen einen signifikanten Unterschied (Tab. 8).

Tab. 4. Wiederfindung von Digoxin mit dem ELISA.

Zu je $2 \mathrm{ml}$ eines Pool-Serums wurden je $1 \mathrm{ml}$ Digoxin-Lösung $(0,07 \mathrm{~mol} / 1$ Phosphatpuffer pH 7,4) hinžugefügt. Jede Konzentration wurde 6-fach bestimmt.

\begin{tabular}{|c|c|c|c|c|c|c|c|c|}
\hline Mąterial & $\begin{array}{l}\text { Digoxin } \\
\text { zugesetzt } \\
(\mu \mathrm{g} / \mathrm{l})\end{array}$ & (nmol/1) & $\begin{array}{l}\text { Digoxin } \\
\text { gefunden } \\
(\mu \mathrm{g} / \mathrm{l})\end{array}$ & (nmol/1) & $\begin{array}{l}\text { Digoxin } \\
\text { gefunden - L } \\
(\mu \mathrm{g} / \mathrm{l})\end{array}$ & $\begin{array}{l}\text { Leerwert } \\
\text { (nmol/1) }\end{array}$ & $\begin{array}{l}\text { Wieder- } \\
\text { findung } \\
(\%)\end{array}$ & $\begin{array}{l}\text { VK}_{\text {Serie }} \\
\text { (\%): }\end{array}$ \\
\hline $\begin{array}{l}\text { Pool-Serum } \\
\text { Pool-Serum } \\
\text { Pool-Serum } \\
\text { Pool-Serum }\end{array}$ & $\begin{array}{l}0 \\
1,0 \\
2,0 \\
3,0\end{array}$ & $\begin{array}{l}(0) \\
(1,28) \\
(2,56) \\
(3,84)\end{array}$ & $\begin{array}{l}0,20 \\
1,24 \\
2,32 \\
3,16\end{array}$ & $\begin{array}{l}(0,26) \\
(1,59) \\
(2,97) \\
(4,05)\end{array}$ & $\begin{array}{l}0 \\
1,04 \\
2,12 \\
2,96\end{array}$ & $\begin{array}{l}0 \\
(1,33) \\
(2,71) \\
(3,79)\end{array}$ & $\begin{array}{r}- \\
104 \\
106 \\
99\end{array}$ & $\begin{array}{l}- \\
17 \\
12 \\
12\end{array}$ \\
\hline
\end{tabular}


Tab. 5. Statistischer Vergleich der Ergebnisse von DigoxinBestimmungen mit RIA und ELISA (13). Patienten ohne Zusatz-Medikation.

1. Vorzeichen-Test nach Dixon \& Mood für Paardifferenzen:

$\mathrm{N}=116 \quad \mathrm{~N}_{+}=58 \quad \mathrm{~N}_{-}=56 \quad \mathrm{~N}_{=}=2 \quad \mathrm{f}=113$

Kein signifikanter Unterschied.

2. Prüfung der Paardifferenzen (d) mit dem t-Test nach Student:
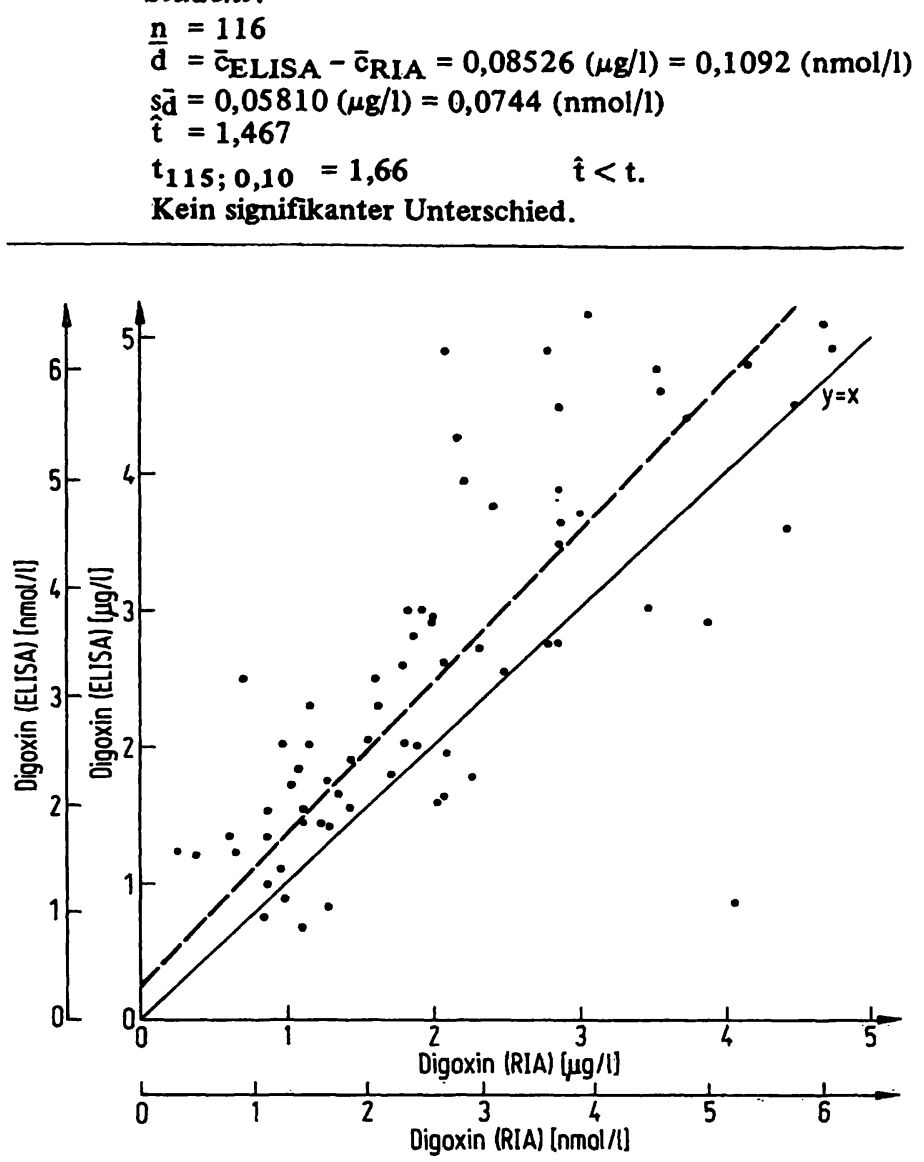

Abb. 3. Vergleich der Ergebnisse von Digoxin-Bestimmungen bei Patienten, die zusätżlich Spironolacton einnahmen.

Ausgezogene Linie: Winkelhalbierende.

Gestrichelte Linie: mittlere Regressionsgerade

Steigung der Regressionsgeraden

Achsenabschnitt der Regressionsgeraden

Korrelationskoeffizient

$\mathrm{n}=68$

$=1,123$

$=0,12 \mu \mathrm{g} / 1$

$[=0,154$

nmol/1]

$=0,714$

\section{Störungen}

Eine Serie von Kontrollseren (TRI-RAC, Dade) ergab eine totale Hemmung der Enzymaktivität. Die Struktur des Konservierungsmittels ist aus den Unterlagen nicht ersichtlich.

\section{Diskussion}

An eine Bestimmungsmethode von Digoxin im Serum zur Therapiekontrolle sind relativ hohe Anforderungen in Bezug auf Nachweisempfindlichkeit und Spezifität zu stellen.

Die ausführliche Erstbeschreibung des von uns geprüften heterogenen Enzymimmunassays durch seine Autoren steht noch aus (vgl. 1.c. (11)). Infolgedessen sind Angaben über die biochemischen Spezifikationen, z. B. die Art der Antikörper-Erzeugung oder die Parameter der Antigen-Antikörperreaktion, unvollständig. Der Test wird als Fertigpackung seit dem August 1977 offiziell im Handel angeboten (15). Unsere Erfahrungen beziehen sich auf Reagenzien, die uns freundlicherweise vor der Freigabe zur Verfügung gestellt wurden und sich möglicherweise vom endgültigen Produkt noch etwas unterscheiden können. Es interessierte uns vor allem die Frage, inwieweit der heterogene Enzym-Immunassay (ELISA) in Bezug auf Zuverlässigkeit und Praktikabilität dem derzeit bei uns routinemäßig verwendeten Radioimmunassay (RIA) gleichwertig ist. Beide Tests benutzen die sog. solid phase Technik, unterscheiden sich jedoch in der Hapten-Indikatorbindung und in der Indikator-Reaktion.

\section{Zuvèrlässigkeit}

\section{Nachweisgrenze und Meßbereich}

Die Nachweisempfindlichkeit und der Meßbereich genügen den klinischen Ansprüchen. Eine Vorverdünnung der Serumprobe ist nur selten erforderlich. Die Indikatorreaktion des ELISA liefert gut meßbare Signale bei stabilem Untergrund.

Tab. 6. In vitro Kreuzreaktion von Digịtoxin.

\begin{tabular}{|c|c|c|c|c|c|}
\hline Material & $\begin{array}{l}\text { Digitox } \\
(\mu \mathrm{g} / \mathrm{l})\end{array}$ & $\begin{array}{l}\text { etzt } \\
\text { (nmol/1) }\end{array}$ & $\begin{array}{l}\text { „Digoxin-- } \\
\text { gefunden } \\
(\mu \mathrm{g} / \mathrm{l})\end{array}$ & (nmol/l) & $\begin{array}{l}\text { Kreuzreaktion } \\
(\%)\end{array}$ \\
\hline 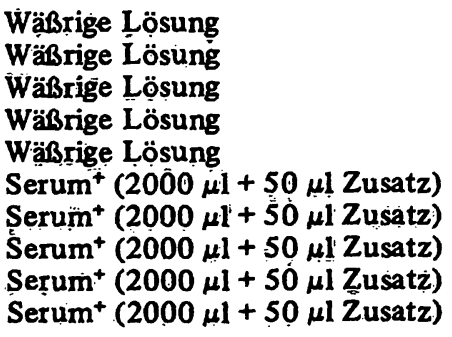 & $\begin{array}{r}9,75 \\
14,63 \\
19,51 \\
24,29 \\
34,15 \\
9,75 \\
14,63 \\
19,51 \\
24,39 \\
34,15\end{array}$ & $\begin{array}{l}(12,7) \\
(19,1) \\
(25,5) \\
(31,8) \\
(44,6) \\
(12,7) \\
(19,1) \\
(25,5) \\
(31,9) \\
(44,6)\end{array}$ & $\begin{array}{l}1,40 \\
1,79 \\
2,22 \\
2,65 \\
2,49 \\
1,24 \\
1,62 \\
1,83 \\
1,85 \\
2,35\end{array}$ & $\begin{array}{l}(1,79) \\
(2,29) \\
(2,84) \\
(3,39) \\
(3,19) \\
(1,59) \\
(2,07) \\
(2,34) \\
(2,37) \\
(3,01)\end{array}$ & $\begin{array}{r}14,4 \\
12,3 \\
11,4 \\
10,9 \\
7,3 \\
12,7 \\
11,1 \\
9,4 \\
7,8 \\
6,9\end{array}$ \\
\hline
\end{tabular}

+ Das Serum war ein Gemisch von 5 Șeren gesunder Perșoṇen von unter 30 Jahren ohne nachweisbaren Digoxin-Gehalt. 
Tab. 7. In vivo Kreuzreaktion von Digitoxin.

In Seren von Patienten, die Digitoxin eingenommen hatten, wurde neben Digitoxin auch Digoxin mit RIA und ELISA bestimmt.

\begin{tabular}{|c|c|c|c|c|c|c|c|c|}
\hline Proben-Nr. & $\begin{array}{l}\text { Digitoxin } \\
\text { RIA } \\
(\mu \mathrm{g} / \mathrm{l})\end{array}$ & (nmol/1) & $\begin{array}{l}\text { Digoxin } \\
\text { RIA } \\
(\mu \mathrm{g} / \mathrm{l})\end{array}$ & (nmol/1) & $\begin{array}{l}\text { "Kreuz- } \\
\text { Reaktion" } \\
\text { des RIA } \\
(\%)\end{array}$ & $\begin{array}{l}\text { Digoxin- } \\
\text { ELISA } \\
(\mu \mathrm{g} / 1)\end{array}$ & $(\mathrm{nmol} / \mathrm{l})$ & $\begin{array}{l}\text {,Kreuz- } \\
\text { Reaktion“ } \\
\text { des ELISA } \\
(\%)\end{array}$ \\
\hline $\begin{array}{l}543 \\
534 \\
531 \\
509 \\
523 \\
497 \\
516 \\
569 \\
570 \\
571 \\
651 \\
661 \\
680 \\
683 \\
684 \\
685 \\
686 \\
689 \\
049 \\
047 \\
048\end{array}$ & $\begin{array}{l}37,6 \\
25,4 \\
18,0 \\
15,7 \\
10,1 \\
10,2 \\
12,3 \\
17,3 \\
12,4 \\
18,7 \\
15,7 \\
17,1 \\
18,9 \\
19,5 \\
12,2 \\
10,7 \\
12,8 \\
19,1 \\
14,2 \\
17,7 \\
13,8\end{array}$ & $\begin{array}{l}(49,1) \\
(33,2) \\
(23,5) \\
(20,5) \\
(13,2) \\
(13,3) \\
(16,1) \\
(22,6) \\
(16,2) \\
(24,4) \\
(20,5) \\
(22,4) \\
(24,7) \\
(25,5) \\
(15,9) \\
(14,0) \\
(16,7) \\
(25,0) \\
(18,6) \\
(23,1) \\
(18,0)\end{array}$ & $\begin{array}{l}1,49 \\
1,07 \\
0,84 \\
0,95 \\
1,56 \\
0,61 \\
0,57 \\
0,81 \\
1,96 \\
0,71 \\
0,62 \\
0,45 \\
0,63 \\
1,44 \\
0,84 \\
0,53 \\
0,48 \\
0,67 \\
0,57 \\
0,57 \\
0,53\end{array}$ & $\begin{array}{l}(1,91) \\
(1,37) \\
(1,08) \\
(1,22) \\
(2,00) \\
(0,78) \\
(0,73) \\
(1,04) \\
(2,51) \\
(0,91) \\
(0,79) \\
(0,58) \\
(0,81) \\
(1,84) \\
(1,08) \\
(0,68) \\
(0,61) \\
(0,86) \\
(0,73) \\
(0,73) \\
(0,68)\end{array}$ & $\begin{array}{r}4,0 \\
4,2 \\
4,7 \\
6,0 \\
15,4 \\
6,0 \\
4,6 \\
4,7 \\
15,8 \\
3,8 \\
4,0 \\
2,6 \\
3,3 \\
7,4 \\
6,9 \\
5,0 \\
3,8 \\
3,5 \\
4,0 \\
3,2 \\
3,8\end{array}$ & $\begin{array}{l}4,32 \\
3,82 \\
2,81 \\
3,37 \\
2,88 \\
2,48 \\
3,38 \\
3,58 \\
5,00 \\
4,00 \\
2,29 \\
2,56 \\
2,50 \\
2,90 \\
2,56 \\
2,37 \\
2,10 \\
2,49 \\
3,17 \\
2,96 \\
2,76\end{array}$ & $\begin{array}{l}(5,53) \\
(4,89) \\
(3,60) \\
(4,32) \\
(3,69) \\
(3,18) \\
(4,33) \\
(4,58) \\
(6,40) \\
(5,12) \\
(2,93) \\
(3,28) \\
(3,20) \\
(3,71) \\
(3,28) \\
(3,03) \\
(2,69) \\
(3,19) \\
(4,06) \\
(3,79) \\
(3,53)\end{array}$ & $\begin{array}{l}11,5 \\
15,0 \\
15,6 \\
21,5 \\
28,5 \\
24,3 \\
27,6 \\
20,7 \\
40,3 \\
21,9 \\
14,6 \\
15,0 \\
13,2 \\
14,9 \\
21,0 \\
22,1 \\
16,4 \\
13,0 \\
22,3 \\
16,7 \\
20,0\end{array}$ \\
\hline
\end{tabular}

Tab. 8. Statistischer Vergleich der Ergebnisse von Digoxin-Bestimmungen mit RIA und ELISA (13). Patienten mit zusätzlicher Medikation von Spironolacton.

1. Vorzeichen-Test nach Dixon \& Mood für Paardifferenzen:

$\mathrm{N}=68 \quad \mathrm{~N}_{+}=53 \quad \mathrm{~N}_{-}=15$

Schranke $(0.5 \%)=26$

$\mathrm{p}<0,005$ Unterschied signifikant

2. Prüfung der Paardifferenzen (d) mit dem t-Test nach Student:

$\underline{n}=68$

$\overline{\mathrm{d}}=\overline{\mathrm{c}}_{\mathrm{ELISA}}-\overline{\mathrm{c}}_{\mathrm{RIA}}=0,48029(\mu \mathrm{g} / \mathrm{l})=0,62(\mathrm{nmol} / \mathrm{l})$

$s_{\bar{d}}=0,10060(\mu \mathrm{g} / \mathrm{l})=0,13(\mathrm{nmol} / \mathrm{l})$

Mittelwerte:

$\begin{aligned} & \overline{\mathrm{C}}_{\mathrm{RIA}}=2,06(\mu \mathrm{g} / \mathrm{l}) ; \\ &(=2,64 \mathrm{nmol} / \mathrm{l})\end{aligned}$

$$
\overline{\mathrm{C}}_{\text {ELISA }}=2,54(\mu \mathrm{g} / \mathrm{l})
$$

$\hat{\mathrm{t}}=\frac{\overline{\mathrm{d}}}{\mathrm{s}_{\overline{\mathrm{d}}}}=4,774$

$t_{67 ; 0,01}=2,65$

$\mathrm{p}<0.01$ Unterschied signifikant

\section{Präzision}

Für ein gleiches Kontrollmaterial ist die Präzision in Serie des ELISA etwas ungünstiger als die Präzision in Serie des RIA (Tab. 1). Dagegen ist die Präzision von Tag zu Tag bei beiden Methoden gleich (Tab. 2) und deckt sich mit Angaben einer Ubersicht über 24 Fertigreagenzien zur Bestimmung von Digoxin durch Radioimmunassays (16). Bei zunehmender Qualitätssteigerung des ELISA dürften Doppelbestimmungen ausreichen. Für klinische Bedürfnisse wäre eine Verbesserung der Präzision wegen der geringen therapeutischen Breite des Digoxins sicher wünschenswert.

\section{Richtigkeit}

Eine anerkannte Referenzmethode existiert nicht. Die Angaben über die Digoxin-Konzentration in Kontrollseren schwanken je nach der verwendeten Methode beträchtlich. Aus dieser Sicht muß die in Tabelle 2 und 3 angegebene Reproduktion von Sollwerten als befriedigend angesehen werden. Die Wiederfindung von Digoxin in proteinhaltigen Lösungen war ebenfalls befriedigend. Unter dieser og. Restriktion kommt einem MethodenVergleich mit klinischem Untersuchụngsmaterial erhöhte Bedeutung zu (vgl. Abb. 2). Die kollektive Übereinstimmung von ELISA- und RIA-Ergebnissen muß als befriedigend bezeichnet werden. Es gibt keinen statistisch gesicherten Unterschied zwischen beiden Ergebnisgruppen. Nicht zu übersehen sind jedoch einige deutliche Ausreißer, für die wir keine befriedigende Erklärung haben. Soweit noch Untersuchungsmaterial vorhanden war, ließen sich diese Abweichungen bei wiederholter Bestimmung mit beiden Methoden bestätigen.

\section{Spezifität}

Der anzustrebende therapeutische Bereich von PlasmaDigoxin liegt bei 0,6 bis $1,5 \mu \mathrm{g} / 1(0,8-1,0$ ñmol/1) (1). Einige ebenfalls klinisch wichtige, in der Struktur ähnliche Substanzen kommen im Serum in wesentlich höheren Konzentrationen vor, so daß schon eine geringe Kreuzreaktivität des Antikörpers eine relevante Störung verursachen könnte. Hinzu kommen u. U. biologisch inaktive Stoffwechselprodukte des Digoxins, die z. T. ebenfalls noch um die Bindungsstellen konkurrieren (17). 


\section{Digitoxin}

Therapeutische Serumkonzentrationen von Digitoxin sollten im Bereich von 9 bis $25 \mu \mathrm{g} / 1$ (12-34 nmol/1) liegen. Die meisten Hersteller von Digoxin-RIA geben eine Kreuzreaktion von Digitoxin an (16). Auch der Digoxin-ELISA zeigt eine Kreuzreaktion für Digitoxin sowohl in vitro wie in vivo. Nach Rietbrock et al. (18) ist bei Digitoxin-Sättigung mit $2 \%(=0,5 \mu \mathrm{g} / \mathrm{l}=0,64$ nmol/1) Digoxin im Serum als Metabolit zu rechnen. Die von uns in vivo gemessene Kreuzreaktion geht über diesen Wert hinaus (Tab. 7). Praktische Bedeutung hat die Kreuzreaktion nur bei Wechsel des Digitalisglykosids oder bei falscher Deklaration des Untersuchungsmaterials.

\section{Spironolacton}

Von größerer Bedeutung ist dagegen die Störung des Digoxin-ELISA durch Spironolacton. 37\% der an uns eingesendeten Proben enthielten gleichzeitig Spironolacton. Nach Sadée et al. (19) können dabei Serumkonzentrationen von Canrenon und Stoffwechselprodukten desselben bis zu $10 \mathrm{mg} / \mathrm{l}$ (etwa $24 \mu \mathrm{mol} / \mathrm{l}$ ) erreicht werden. Selbst unter weniger extremen Konzentrationsverhältnissen wird man mit einem 100fachen Uberschuß der Störsubstanzen rechnen müssen, der bereits bei einer Kreuzreaktion von $1 \%$ zu einer Verdopplung des Ergebnisses der Digoxin-Bestimmung führen würde. Der von uns in vivo festgestellte Effekt ist zwar geringer. Immerhin liegen aber die Digoxin-ELISA Ergebnisse um durchschnittlich $0,48 \mu \mathrm{g} / 1(0,14$ bis $1,14 \mu \mathrm{g} / 1=0,18$ bis $1,46 \mathrm{nmol} / \mathrm{l}$ ) höher als die gleichzeitig bestimmten Digoxin-RIA Konzentrationen (Abb. 3, Tab. 8). Diese Störung dürte im oberen therapeutischen Bereich eine Rolle spielen, wo die Abgrenzung zwischen therapeutischen und toxischen Konzentrationen kritisch ist.

\section{Störungen der Indikatorreaktion}

Während eine Störung durch radioaktives Material in der Probe nicht zu erwarten ist, muß mit Störungen durch Enzyminhibitoren gerechnet werden. In dieser Beziehung sind uns Kontroll-Seren mit deklariertem Digoxin-Wert aufgefallen, die das Indikator-Enzym Peroxidase total inhibierten. Es besteht der Verdacht, daßß es șich hierbei um das viel benuitzte Natriumazid handelt. Störungen durch Hämolyse, Ikterus oder Hyperlipämie sind bisher nicht aufgefallen. Der heterogene Enzymimmunassay dürfte in dieser Beziehung weniger anfällig sein als der homogene Enzymimmunassay.

\section{Praktịkabilität}

Der größte Vorteil des ELISA liegt im Vermeiden des Umgangs mit radioaktiven Substanzen. Für kürzere Serienlängen bis zu 10 Proben genügt für die Messung ein Photometer üblicher Qualität. Der eigentliche Meß- vorgang kann teilweise mechanisiert werden. Der Geräteaufwand erreicht dann allerdings den Preis eines GammaMeßplatzes, so daß hier kein wesentlicher Preisvorteil zu erwarten ist. Die Bezugskurve muß in jeder Serie neu erstellt werden. Im Falle einer Sofort-Analyse bei fraglicher Digitalisüberdosierung erscheint es vertretbar, die Zahl der Bezugspunkte auf 2 (3,0 und 1,5 $\mu \mathrm{g} / 1$ $=3,84$ und $1,92 \mathrm{nmol} / \mathrm{l})$ zu reduzieren. Wird für jede Probe eine Dreifachbestimmung angesetzt, so reicht eine Packung mit 96 Röhrchen für 25 Proben einschließlich Bezugslösungen und Qualitätskontrollen aus. Der Zeitaufwand ohne Teilmechanisierung der Messung beträgt für 25 Proben etwa 3 Stunden, der bei Einsatz eines Dilutors und Protokolldruckers durch Kürzung des Arbeitstaktes noch etwas reduziert werden kann. Bei zunehmender Perfektionierung der Reagenzien und genügennder Erfahrung des Anwenders dürften Doppelbestimmungen ausreichen. Im Gegensatz zum homogenen Enzymimmunassay, der weitgehend mechanisiert werden kann (10) und aus meßtechnischen Gründen auch sollte, ist der heterogene Enzymimmunassay mit den zur Zeit bekannten Analysengeräten nicht vollmechanisierbar. Die Forderung nach Vollmechanisierung steht $u$. E. jedoch angesichts relativ kurzer Serienlängen nicht im Vordergrund. Eine rechnerunterstützte Auswertung analog der Auswertung des RIA $(10,21)$ dürfte einen zusätzlichen Rationalisierungseffekt bieten. Die Kosten pro Test für die Reagenzien des ELISA und des von uns verwendeten RIA liegen in der gleichen Größenordnung.

\section{Schlußfolgerung}

Die Bestimmung von Digoxin im Serim mit einem heterogenen Enzymimmunassay (ELISA) ist für klinische Zwecke brauchbar und bietet damit eine gleichwertige Alternative zur Bestimmung durch einen Radioimmunassay.

\section{Danksagung}

Wir danken Frau $H$. Hartwig für die gewissenhafte Durchführung der Versuche.

\section{Anmerkung bei Korrektur des Manuskriptes}

1. Nach Abschluß der Versuche stellte uns der Hersteller eine neue Antikörper-Charge mit verminderter Kreuzempfindlichkeit gegen Canrenon vor. Bei 21 Patienten, die gleichzeitig Digoxin und Spironolacton einnahmen, konnten wir keine signifikanten Unterschiede zwischen den Ergebnissen des RIA und ELISA mehr feststellen. Dies war auch nicht der Fall in einer weiteren Serie von Patienten, die Aldactone intravenös verabreicht bekamen.

2. Die Firma Dade stellte uns freundlicherweise Kontrollseren ohne Stabilisator-Zusatz zur Verfügung, die nach orientierenden Versuchen im ELISA ohne Störung verwendet werden können. 


\section{Literatur}

1. Rietbrock, N. \& Kuhlmann, J. (1977), Med. Klin. 72, 435-449.

2. Schüren, K. P. \& Rietbrock, N. (1977), Internișt. Prạxis $17,581-601$.

3. Smith, T. W. \& Haber, E. (1970), J. Clin. Invest. 49, 2377-2386.

4. Wisdom, B. (1976), Clin. Chem. 22, 1243-1255

5. Chang, J. J., Crowl, C. P. \& Schneider, R. S. (1975), Clin. Chem. 21, 967.

6. Rosenthal, A. F., Vargas, M. G. \& Klass, C. S. (1976), Clin. Chem. 22, 1899-1902.

7. Sun, L. \& Spiehler, V. (1976), Clin. Chem. 22, 2029-2031.

8. Müller, H., Bräuer, H., Reinhardt, M. \& Förster, G. (1976), Arrtl. Lab. 22, 399-402.

9. Drost, R. H., Plomp, Th. A., Teunissen, A. J., Maass, A. H. J. \& Maes, R. A. A. (1977), J. Clin. Chem. Clin. Biochem. 15, 151.

10. Vogt, W., Tausch, A., Jacob, K. \& Knedel, M. (1977), J. Clin. Chem. Clin. Biochem. 15, 194-195.

11. Kleinhammer, G., Lenz, H., Linke, R. \& Gruber, W. (1976), Europäischer Kongreß für Klinische Chemie, Prag 4. 10. 76, Abstract.
12. Engvall, E., Jonssōn, K. \& Perlmann, P. (1971), Biochim Biophys. Acta $251,427-434$.

13. Sachs, L. (1972), Statistische Methoden. Springer-Verlag, Berlin. 3. Aufl.

14. Averdunk, R. \& Borner, K. (1970), Z. Klin. Chem. Klin. Biochem. 8, 263-268.

15. Fa. Boehringer-Mannheim: Diagnostica Dialog. Heft 3/77. Mannheim.

16. Hopkins, J. A. C., Ëdwards, L., Herner, A. E. \& Van Dreal, P. (1977), Clin. Chem. 23, 403-446.

17. Fläsch, H., Heinz, N. \& Petersen; R. (1977), Arzneimittelforsch./Drug Res. 27, 649-653.

18. Rietbrock, N., Vöhringer, H. F. \& Kuhlmann, J. (1977), Herż und Kreislauf, im Druck.

19. Sadée, W., Schröder, R. ., v. Leitner, E. \& Dagcioglu, M. (1974), Europ. J. Clin. Pharmacol. 7, 195-200.

20. Vogt, W., Popp, B. \& Knedel, M. (1973), Z. Klin. Chem. Klin. Biochem. 11, 438-445.

21. Schöneshöfer, M. (1977), Clinica Chimica Acta 77, 101-115.
Prof. Dr. med. K. Borner Institut für Klinische Chemie und Klinische Biochemie der Freien Universität Berlin, Klinikum Steglitz Hindenburgdamm 30 D-1000 Berlin 45

und

Prof. Dr. N. Rietbrock Institut für Klinische Pharmakologie der Freien Universität Berlin Hinderiburgdamm 30 D-1000 Berlin 45 